\title{
CORPUS Corpus
}

Archivos virtuales de la alteridad americana

Vol 3, No 2 | 2013

Julio / Diciembre 2013

\section{Vizcaínas: un proyecto de conservación, desarrollo social y cultural mexicano con 280 años de historia}

Vizcainas: A conservation, social and cultural development, with 280 years of Mexican history

Ana Rita Valero de García Lascuráin y Nora Deveaux Cabrera

\section{OpenEdition}

\section{Journals}

Edición electrónica

URL: http://journals.openedition.org/corpusarchivos/589

DOI: 10.4000/corpusarchivos.589

ISSN: $1853-8037$

Editor

Diego Escolar

\section{Referencia electrónica}

Ana Rita Valero de García Lascuráin y Nora Deveaux Cabrera, « Vizcaínas: un proyecto de

conservación, desarrollo social y cultural mexicano con 280 años de historia », Corpus [En línea], Vol 3, No 2 | 2013, Publicado el 20 diciembre 2013, consultado el 22 abril 2019. URL : http:// journals.openedition.org/corpusarchivos/589; DOI : 10.4000/corpusarchivos.589

Este documento fue generado automáticamente el 22 abril 2019. 


\section{Vizcaínas: un proyecto de conservación, desarrollo social y cultural mexicano con 280 años de historia}

Vizcainas: A conservation, social and cultural development, with 280 years of

Mexican history

Ana Rita Valero de García Lascuráin y Nora Deveaux Cabrera

\section{México de papel}

1 Hablar sobre la historia de un país es remitirse a sus fuentes históricas y México no es la excepción. Este país es rico en fuentes documentales, las cuales abarcan todas las épocas de su desarrollo histórico, encontrando así archivos que contienen información tan antigua que hace referencia a la fundación de la ciudad por las culturas prehispánicas como documentos del siglo XVI hasta el siglo XXI.

2 En algunos archivos encontraremos los códices que son documentos pictográficos elaborados por tlacuilos ${ }^{1}$. Estos manuscritos relatan el desarrollo de Mesoamérica hasta la llegada del capitán Hernán Cortés con su campaña militar, su posterior conquista y colonización, para dar paso a la fundación del Virreinato de la Nueva España.

La época colonial para México abarcó un periodo de 300 años. La Nueva España generó millones de documentos que tenían como fin dar órdenes, avisos, implementar nuevas acciones legales y administrativas; asimismo, libros de cuentas diversas, libros de juntas de cabildo, constituciones de colegios, registros sobre el nacimiento y muerte la de población, asuntos fiscales, recetas médicas, acreditaciones de grado, calificaciones, silabarios, mapas, planos, partituras de música sacra, entre otros. Dicha documentación ha sido reunida en los archivos con la finalidad de resguardarla, conservarla y difundir su riqueza ${ }^{2}$. 
4 La época independiente dejo miles de testimonios en papel, como: administración de aduanas, alcabalas, regimiento de infantería del cuarto batallón, cargo y data de la Real Hacienda, cargo y data de papel sellado, mercedes, licencias para ordenación arzobispal, censos, libros de cuentas de colegios y hospitales, consolidación de vales reales, reales decretos, pasaportes de navegación mercantil, entre otros.

Mencionamos este proceso del siglo XIX por ser el inicio de una nueva etapa de organización para nuestro país, el cual se encuentra inmerso en este siglo. Es un periodo lleno de reorganización, ya que en él se darán las bases de lo que es México en la actualidad. Como herencia de este largo periodo tenemos documentación que hace referencia a Juntas Menores Auxiliares, asuntos relacionados con la beneficencia pública y privada, cárceles y penitenciarias, casas de empeño, censos y estadísticas, Colegio de Minería, Colonia Penal, colonización, Congreso General, Consejo de Gobierno, correos, vapores, diario oficial de la federación, impresos oficiales, imprenta del gobierno, división territorial y límites entre los Estados, elecciones, epidemias, festividades nacionales, inspección sanitaria, instrucción pública, jardín botánico, jefes políticos, legislaturas de los Estados, loterías, rifas, milicias nacionales, misiones, panteones, pasaportes, policía, presos, registro civil, relaciones con los Estados, salubridad, tabaco, prontuarios, leyes, reglamentos y circulares, por mencionar algunos eventos históricos.

6 De la época moderna y de la época contemporánea podríamos destacar toda la documentación que generaron las secretarías del país, por ejemplo: Secretaría del Trabajo y Prevención Social, agrupaciones obreras, Compañía Mexicana de Petróleos "El Águila", Secretaría de Agricultura, Administración de Bienes Nacionales, Dirección General de Ganadería, Dirección General de Comercio, Dirección General de Sanidad Animal, Dirección de Cría y Fomento Pecuario, Subsecretaría de Ganadería, Dirección General de Comercio, Centro Nacional Avícola, Secretaría de Relaciones Exteriores, Comisión de Aguas del Valle de México, entre otros.

7 Hemos mencionado algunas fuentes considerando que es útil para el lector interesado en conocer y acercarse a este tipo de documentación ya que nos permite analizar y presentar una reconstrucción parcial de la realidad histórica de nuestros antepasados, y así entender qué sucede en nuestro presente.

\section{Vizcaínas: 280 años de historia}

Iniciaremos nuestro artículo tomando como contexto la época novohispana. En este periodo se funda e inicia sus actividades la institución que hoy queremos presentar a los lectores de la Revista Corpus: el Archivo Histórico "José María Basagoiti Noriega", del Colegio de San Ignacio de Loyola, Vizcaínas. Este se encuentra ubicado en el corazón de la Ciudad de México, el centro histórico en la calle de Vizcaínas 21, colonia Centro, delegación Cuauhtémoc, c.p. 06080.

La edificación del colegio se debe a un grupo de vascongados, miembros de la cofradía de Nuestra Señora de Aránzazu ${ }^{3}$ quienes se percataron de que las escuelas de amigas, los conventos y colegios que prestaban los servicios de enseñanza estaban rebasados en su capacidad para recibir y atender a la población femenina novohispana en edad de recibir enseñanza, ${ }^{4}$ provocando con ello el abandono de los lustros del pundonor y una relajación total de costumbres, sirviendo así de notables escándalos, perniciosos ejemplos y la ruina de la pública honestidad. ${ }^{5}$ 
10 En una junta de cabildo, los vascongados decidieron esbozar un proyecto para construir un inmueble que tendría la capacidad de albergar a una población femenil de quinientas colegialas. Todos los gastos generados para la fabricación y organización del colegio fueron subsidiados únicamente con los capitales de los cofrades.

11 Así la logística y organización de dicha obra iniciaría en el año de 1731. Al año siguiente, se recabarían los capitales y materiales necesarios para llevar a cabo la obra. En 1734, el virrey don José Antonio de Vizarrón y Eguiarreta aprueba la fundación y otorga en merced el solar donde se realizaría su construcción. En ese mismo año, se coloca la primera piedra. El arquitecto fue José de Rivera, los materiales utilizados para su elaboración fueron tezontle ${ }^{6}$ y cantera ${ }^{7}$. Su extensión es de 100 metros cuadrados y constituye una manzana entera ${ }^{8}$. La construcción de este edificio se extendería por 33 años y llegaría a su fin en el año de 1767.

12 En el interior del colegio la distribución consta de una planta baja y un primer piso, en los cuales se distribuyeron las viviendas para las colegialas, una capilla, seis patios y un huerto. Sus cuatro fachadas están constituidas por accesorias de taza y plato9. Sus rentas generarían ingresos al colegio para ayudar a la manutención del inmueble y de las colegialas. Cabe mencionar que en la actualidad varias de estas accesorias son rentadas para dar servicio al público en los rubros papelería y café, y servir de sede a AMABPAC ${ }^{10}$.

13 La apertura del Real Colegio de San Ignacio de Loyola fue un acto solemne, al cual asistieron las autoridades del virreinato, los miembros de la cofradía y con ellos las primeras colegialas que estarían internas en la institución. La dirección del Colegio estaría a cargo de la mesa de la Cofradía; sus integrantes votarían por decisión unánime lo referente a lo relacionado con lo administrativo, económico y qué alumnas serían dotadas, así como las personas que atenderían la dirección interna del colegio conformada de la siguiente manera:

Administración: rectora, vice-rectora, secretaria

Enseñanza: primera de vivienda

Salud: médico, una enfermera

Clausura de la institución: porteras, torneras, escuchas, celadoras

Servicios religiosos: sacristán mayor, primer capellán, segundo capellán, sacristana, prefecta de coro

La educación en San Ignacio era una formación cristiana en combinación con las labores de manos. Las primeras de vivienda eran las encargadas de impartir esta enseñanza; sus materiales de apoyo estaban conformados especialmente por:

* Silabario o cartilla. Se empleaba para el aprendizaje de la lectura y el deletreo, ya que contenía sílabas, dicciones y el arte y modo de hacer las lecturas. A la vez mostraba los diferentes tipos de versos tales como endecasílabos, endechas, redondillas, cuartetas, sonetos, décimas, etcétera ${ }^{11}$.

* Tinta, mango, punta y papel, para el aprendizaje de la escritura.

* Tablas matemáticas

* Compendio matemático de Benito Blas o de Puig Mora

* Tratado de aritmética elemental realizado por Juan Claudio Díaz y Esteban Morea. ${ }^{12}$

* Catecismo del Padre Jerónimo Ripalda. ${ }^{13}$

* Catecismo Histórico que contiene en resumen la historia santa y la doctrina cristiana elaborada por el padre Claudio Fleury. ${ }^{14}$

* El Año Cristiano o Ejercicios devotos. 
* Los Ejercicios Espirituales de San Ignacio de Loyola. ${ }^{15}$

Los siguientes compendios religiosos servirían para que las doncellas aprendieran la doctrina que era enseñada conjuntamente con la lectura y las primeras oraciones memorizadas con el silabeo. Cabe mencionar que las dos últimas obras eran para las colegiadas de los grados más avanzados. clavicordio y la flauta como parte de su enseñanza musical -la cual fue impartida en la escoleta del colegio-y recibían instrucciones para el canto (Muriel, 2006, pp. 59-60).

A su vez, las alumnas de Vizcaínas aprenderían las labores de manos, instrucción que se daba en la sala de labor. Aquí se aprendía todo lo referente a la costura desde las primeras puntadas ${ }^{16}$ que serían practicadas en un dechado. ${ }^{17}$ Una vez dominadas estas primeras puntadas continuaban con el zurcido y el remiendo, para dar paso al último elemento de la labor, el más fino y delicado: el bordado, actividad que constaba de varias características que las hacian exquisitas a simple vista. ${ }^{18} \mathrm{El}$ bordado llevaba una combinación de hilos de origen natural con los de origen metálico, elementos que ofrecieron diversos matices en los diferentes diseños; además, para embellecer estos delicados trabajos se utilizó pedrería. Una vez aprendido el bordado en color la siguiente labor a realizar era el bordado en blanco. ${ }^{19}$

8 La distribución de la enseñanza se regía por horarios. Las actividades iniciaban a las cinco treinta de la mañana; se levantaban y alistaban para escuchar misa a las seis en el coro bajo; regresaban a sus viviendas; terminaban las tareas a las nueve y hasta las doce atendían a las costuras, labores, bordados, lectura y escritura, "o semejantes honestos ejercicios" ${ }^{20}$; regresaban a su vivienda para comer, después "podrán dormir la siesta si lo desean". Posteriormente "al coro para rezar el rosario o corona de nuestra señora la santísima Virgen María con sus letanías, [...] los lunes, miércoles y viernes que no sean festivos lo realizarán a puerta cerrada y sin luz, después realizarán sus novenas o devociones particulares, el horario en inverno será hasta las siete y media y en verano hasta las ocho y media". ${ }^{21} \mathrm{Al}$ terminar los ejercicios cristianos regresban a sus viviendas para cenar y a las nueve se escuchaba la campana que indicaba la hora de dormir.

Lo que hemos mencionado en líneas anteriores es la vida cotidiana del colegio. Sin embargo debemos resaltar que Vizcaínas tendrá la gran virtud de ser la única institución que prestará sus servicios de forma ininterrumpida desde su fundación hasta la actualidad. Constituye, de esta manera, uno de los establecimientos longevos que han vivido el desarrollo de todos los procesos de la historia de México. Todos y cada uno de ellos han realizado transformaciones en el colegio. Por mencionar algunos indicaremos que después del proceso de independencia frente a la Corona española, la administración económica estuvo severamente afectada. En la guerra de 1847 de México contra Estados Unidos la institución prestó una parte de sus instalaciones para establecer un banco de sangre. Su etapa más difícil fue en 1859, cuando el Presidente Benito Juárez decreta la aplicación de la Ley de Nacionalización de Bienes eclesiásticos. A grandes rasgos esta ley decretaba que todos los bienes de la Iglesia pasaran a manos del Estado. El Colegio se salvó de la desamortización porque los cofrades vascos explicarían al Ministro de Hacienda, Melchor Ocampo, que la institución se encargaba exclusivamente de resguardar y educar a la población femenina precisada de ayuda y que sus fondos eran totalmente conseguidos a través de las donaciones de sus bienhechores y sus rentas particulares. 

resultados serían que el colegio incorporaría a su educación nuevas clases, como la clase de gimnasia; con ello se trataba de dar una educación integral en que se desarrollase tanto el cuerpo como la inteligencia. A su vez los trabajos manuales también recibieron gran impulso, procurando que la enseñanza tuviese un carácter útil para la sociedad. El Colegio en este periodo se consolidó como uno de las instituciones de mayor prestigio de la ciudad de México. ${ }^{22}$

21 En la Revolución Mexicana y los años siguientes, los acontecimientos que afectaron al colegio fueron económicos, los cuales hicieron que la institución tuviera que ajustarse en sus gastos para continuar con su labor educativa. Finalmente, el siglo XX y XXI serían para Vizcaínas periodos de cambios, tanto en su arquitectura interna como en sus planes de estudio para poder adaptarse a las nuevas necesidades de la niñez mexicana. En la actualidad el colegio imparte educación básica (kinder, primaria, secundaria) y educación media (preparatoria).

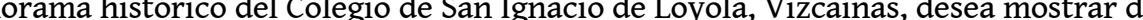
forma general cómo la institución amparó, protegió y promovió el desarrollo de la educación de la mujer para que desempeñara su rol social de madre y esposa; sin olvidar su preparación para su inclusión en el desarrollo económico del país al adiestrarla en artes y oficios acordes a su sexo. Nuestra aportación es interesar a los lectores a que descubran las riquezas documentales que resguarda su archivo.

\section{Conservación y desarrollo de la memoria histórica de Vizcaínas}

Una vez que Vizcaínas inicia su actividad educativa, paralelamente inicia su archivo. El cofrade don Ambrosio de Meave establece el cuidado y la organización de todos y cada uno de los documentos generados por el colegio. A él se le atribuye la fundación de este repositorio.

Su posterior cuidado y desarrollo se realizó con el interés, trabajo y cuidado que le dio su mecenas don José María Basagoiti y Noriega, quien fue presidente del Consejo de Patronos Honorarios y Presidente Honorario de la Mesa Directiva del Colegio. En homenaje a su labor de conservación y a su gran interés y amor a la memoria histórica el archivo lleva su nombre.

Junto a su trabajo se sumarían múltiples esfuerzos de varias personas que han continuado su misión a lo largo de los siglos, como la centena de rectores de la Mesa de Aránzazu y los presidentes de la Junta Directiva, así como todas y cada una de las rectoras y directoras del colegio.

Más adelante, en las postrimerías del siglo XIX, el historiador don Enrique Olavarría y Ferrari ordenaría por primera vez los fondos del archivo con un interés plenamente académico; la intención era escribir la historia del Colegio, obra que se publicaría en 1889 bajo el título de El Real Colegio de San Ignacio de Loyola, vulgarmente Colegio de las Vizcaínas, en la actualidad Colegio de la Paz. Reseña histórica.

27 En la época de Olavarría se guardaban las colecciones en un salón de la planta baja. Sin embargo este repositorio era inadecuado por la humedad y temperatura prevaleciente; por ello muchos documentos comenzaron a deteriorarse y corrían el peligro de

Corpus, Vol 3, No 2 | 2013 
desaparecer. Por ello, algunos años después, se tomó la decisión de trasladarlos a la planta alta con una primera idea de conservación. Afortunadamente, algunas décadas después, don Pedro Lascuráin, presidente de la Junta Directiva del Colegio, mandó arreglar el archivo para continuar la "reseña histórica" que había escrito Olavarría y Ferrari. Lascuráin encomendó dicha tarea a Julia Mörner, antigua alumna y directora de Vizcaínas, quien ayudada por Soledad Salazar trabajaría bajo la tutela de Francisco Fernández del Castillo, eminente historiador e investigador oficial del Archivo General de la Nación. Él quedó como primer director del archivo histórico del colegio, cargo durante el cual comenzó a estructurar los documentos en legajos y libros de las colecciones allí resguardados; a su muerte, ocurrida en 1936, seguiría Julia Mörner colaborando en tan importante repositorio.

Un par de años después, hacia 1938, con el objeto de crear el museo del Colegio, se le encomendó a Gonzalo Obregón, historiador de arte, el cuidado del archivo; este seguramente siguió ordenando los legajos, ayudado por algún tiempo todavía por Julia Mörner.

30 Sobresale en la trayectoria del archivo la presencia de la historiadora Josefina Muriel, quien volvió a organizar una buena parte de los documentos originales. Las técnicas archivísticas contemporáneas fueron introducidas al archivo gracias al trabajo de Cristina García Vallejo, quien automatizó los fondos para lograr así un mejor aprovechamiento de la información. Cabe señalar que tanto Olavarría y Ferrari como Gonzalo Obregón, Julia Mörner y Josefina Muriel no solo se ocuparon del cuidado de las colecciones, sino que paralelamente escribieron la historia de la institución.

31 Siguiendo con la tarea de conservar y difundir dicha memoria documental se han emprendido varios proyectos. Uno de ellos fue el trabajo en conjunto con Ayuda al Desarrollo de Archivos Iberoamericanos (ADAI) para catalogar el fondo más antiguo del archivo: la Archicofradía del Santísimo Sacramento y Caridad. El archivo recibió el título de Memoria del Mundo por su fondo Colegio de San Ignacio de Loyola. También se realizan trabajos de fumigación y catalogación constantes, los cuales buscan mejorar la consulta y ordenación del archivo, el cual cuenta con una colección documental de 2.000 volúmenes, conformados por legajos y libros, concentrados en ocho fondos documentales de un gran valor, ya que nos ofrecen rica información detallada de su vida cotidiana.

Cabe señalar que dichos fondos no se acotan exclusivamente a los que ha generado el Colegio de San Ignacio a lo largo de su trayectoria pedagógica. Su riqueza documental se complementó en el siglo XIX, cuando tuvo que albergar y hacerse cargo de las instituciones que fueron suprimidas como resultado de la aplicación de las Leyes de Reforma, tales como documentos de tres de las cofradías más influyentes que se desarrollaron en el México virreinal: La Cofradía de Nuestra Señora de Aránzazu que concibió, proyectó y financió el Colegio de San Ignacio de Loyola; la Archicofradía del Santísimo Sacramento y Caridad, que tuvo bajo su patrocinio el Colegio de Niñas de la Caridad (en el ámbito educativo) y la Congregación del Divino Salvador del Mundo y Buena Muerte (en el ámbito de la sanidad); y por último el Recogimiento Colegio de Niñas de San Miguel de Belém.

El archivo cuenta también con el fondo documental de la Real Sociedad Vascongada de Amigos del País, conformada por cerca de 500 novohispanos de origen vascuence, asentados a todo lo largo del virreinato. La última sección contiene una serie de métodos y obras musicales usadas en los colegios como parte importante de los programas 
educativos y ceremoniales dirigidos a la mujer; así como el nuevo fondo llamado Fondo Patronato, con los documentos provenientes de los miembros de Patronato de Vizcaínas y en su caso los de sus empresas.

En añadidura a los fondos documentales, el archivo resguarda también una biblioteca conformada por las piezas originales usadas en la enseñanza del Colegio de San Ignacio durante el siglo XIX, así como cuatro colecciones más; una de ellas, de carácter religioso, procede del antiguo Convento de San José de Gracia; otra formada por las obras históricas publicadas en España, y una tercera, de reciente adquisición, formada por los libros de la Sociedad de Amigos del País, con temas de la cultura vasca, que incluyen arte, historia, economía, política, genealogía y tolo lo relacionado a los asuntos vascuences. A esta gran colección se une la nueva Biblioteca José María Basagoiti Noriega, que ingresó al archivo recientemente; está formada por obras de diferentes temas entre los que se cuentan historia, filosofía, teología, política y arte entre otros temas.

La conjunción de dichas colecciones resguardadas actualmente en el Archivo Histórico del Colegio de San Ignacio de Loyola, Vizcaínas, refleja la presencia de un importante proyecto de carácter social, que se llevó a cabo en la Nueva España desde los tempranos años del arranque colonial y que hoy orgullosamente continúa de forma ininterrumpida.

Por todo lo anterior, con el objetivo de dar a conocer todas las riquezas de las fuentes documentales con las que contamos, deseamos informar a los especialistas que la documentación del acervo está clasificada en forma topográfica de la manera siguiente: estante, tabla, volumen y expediente. Se encuentra ordenada en una base de datos computarizada, por fondo, procedencia, cronología y tema. Dos terceras partes de las colecciones ya se encuentran microfilmadas, gracias a la generosidad del Centro de Estudios de Historia de México Condumex, y queda pendiente aún el resto.

Contamos con los siguientes instrumentos de consulta:

Base de datos elaborada de acuerdo a la clasificación temática, onomástica y cronológica. Guía documental impresa y en soporte electrónico ordenada por clasificación de volúmenes, con explicaciones breves del contenido de cada uno de ellos.

Fichero con clasificación temática de los volúmenes.

Como material de apoyo y de difusión contamos con la venta de los siguientes materiales ${ }^{23}$

Guía documental impresa de la Biblioteca del Convento de San José de Gracia Siglos XVIII y XIX

Guía General en soporte electrónico del catálogo del Archivo

Finalmente queremos reiterar la invitación a los lectores de Corpus a que se acerquen a los fondos que alberga el archivo, para que junto con nosotros sigamos difundiendo la enorme veta de temas que esperan por ser investigados. A la vez agradecemos a la revista este espacio el cual nos permite dar a conocer una parte de la riqueza documental con la que cuenta México. 


\section{BIBLIOGRAFÍA}

Atkinson, F. (1908). Economía e higiene doméstica de Appleton. Arreglada para el uso de la familia en general y para texto en las escuelas y colegios de señoritas. D. Appleton y Compañía, pp. 135,136.

Fleury, C. P. (1884). Catecismo Histórico que contiene en resumen la historia santa y la doctrina cristiana. México: Antigua imprenta de Murguía.

Galarza, J. (1974). Códices mexicanos. Catálogo de la Biblioteca Nacional de París. París: El Museo del Hombre.

Galarza, J. (1980). Doctrina christiana: Un método para el análisis de un manuscrito pictográfico mexicano XVIII ${ }^{\underline{a}}$ siglo con la Oración de la primera aplicación. Pater Noster: Sociedad de Etnología.

Galarza, J. (1986). Amatl, amoxtli, El papel el libro: los códices mesoamericanos. México: Escuela Nacional de Antropología e Historia.

Galarza, J. (1992). Tlacuilo. Il Segreto della Svelato SCRITTURA Azteca. Firenze.

Galarza, J. (1992). En amoxtli en tlacatl, el libro, el hombre. Códices y vivencias. México: Editorial Tava.

Galarza, J. (1996). El color en los códices. México: Editorial amatl.

Galarza, J. (1996). Códices y pinturas tradicionales Indígenas en el Archivo General de la Nación. Catálogo. México: Editoriales amatl Tava-Librería Madero.

Gonzalbo, P. (1985). La educación de la mujer en la Nueva España. México: Ediciones el caballito. Secretaría de Educación Pública.

Gonzalbo, P. (1999). Historia de la educación en la época colonial. La educación de los criollos y la vida urbana. México: El Colegio de México.

Gonzalbo, P. (2001). Educación y colonización en la Nueva España 1521-1821. México: Universidad Pedagógica Nacional.

Loyola, I. (1999). Ejercicios Espirituales. Barcelona, España: Ediciones Abraxas, Océano.

Muriel, J. (1995). Conventos de Monjas en la Nueva España. México: Editorial Jus S. A de C. V.

Muriel, J. (2004). La Sociedad Novohispana y sus Colegios de Niñas. México: Instituto de Investigaciones Históricas. Universidad Nacional Autónoma de México.

Muriel, J. (coordinadora) (2006). Los vascos en México y su Colegio de las Vizcaínas. México: Integración Editorial S. A. de C. V.

Obregón Jr. G. (1949). El Real Colegio de San Ignacio de México. (Las Vizcaínas). México: El Colegio de México.

Olavarrìa y Ferrari, E. (1889). El Real Colegio de San Ignacio de Loyola vulgarmente Colegio de las Vizcaínas en la actualidad Colegio de la Paz. Reseña Histórica. México: Imprenta de Francisco Díaz de León.

Ripalda, J. P. (1863). Catecismo de la Doctrina Cristiana. México: publicado por Mariano Galván Rivera, 1863, sexta edición. 
Torales Pacheco, J. M. C. (2001). Ilustrados en la Nueva España. Los socios de la Real Sociedad Bascongada de los Amigos del País. México: Universidad Iberoamericana- Real Sociedad Bascongada de los Amigos del País-Colegio de San Ignacio de Loyola Vizcaínas, I. A. P.

\section{NOTAS}

1. Tlacuilos es un vocablo náhuatl que denomina a la persona que se encargaba de elaborar las pictografías de los códices. Para más información consultar las obras escritas por Joaquín Galarza.

2. México cuenta con una gran variedad de archivos históricos los cuales resguardan una gran cantidad de fuentes documentales. Por mencionar algunos contamos con el Archivo General de la Nación (AGN), Archivo del Distrito Federal "Carlos Sigüenza y Góngora", Archivo de Notarías de la Ciudad de México, Archivo Histórico del Agua, Archivo Histórico del Arzobispado, Archivo del Cabildo de la Catedral de México, Archivo Histórico del Centro de Estudios de Historia de México CUNDUMEX hoy en día CARSO, Archivo Histórico de Petróleos Mexicanos, Acervo Histórico de Bellas Artes, acervo de concentración e Histórico de la Secretaria de Salud, entre otros.

3. Fundada en una capilla en el convento de San Francisco.

4. Las principales instituciones que se encargarán de educar a las doncellas novohispanas a lo largo de todo el virreinato serán los conventos de monjas; su primer establecimiento se dio en 1536 y fue el Convento de La Concepción, el segundo fue el Convento de Santa Clara, fundado en 1570; posteriormente el Convento Nuestra Señora de la Balvanera, fundado en 1573; el Convento de Regina Coello, fundado en 1573; el Convento de Jesús María, fundado en 1578; el Convento de Santa Catalina de Sena, fundado en 1581; el Convento de San Jerónimo, fundado en 1585; el Convento San Juan de la Penitencia, fundado en 1591; el Convento de la Encarnación, fundado en 1594; el Convento de Santa Isabel, fundado en 1601 y el Convento de San José de Gracia, fundado en 1610. Dentro de este escenario se comenzarían a esbozar y cimentar colegios que se encargarían de educar a las doncellas novohispanas. Las edificaciones que prestaron sus servicios de enseñanza fueron el Colegio de Nuestra Señora de la Caridad en 1548, posteriormente se une a esta labor el Colegio Recogimiento de San Miguel de Belén en el año de 1683. Para el año de 1732 se funda el Colegio de San Ignacio de Loyola Vizcaínas y por último el Colegio de Nuestra Señora del Pilar (La Enseñanza) en 1754.

5. Archivo Histórico "José María Basagoiti y Noriega" del Colegio de San Ignacio de Loyola las Vizcaínas, E5 TV V7, NC 14463, 14464. Para las siguientes citas ocuparemos las siglas AHCV para referirnos al archivo.

6. Tezontle es una piedra volcánica porosa, muy ligera, de color rojo oscuro, usada en construcción.

7. Cantera es una piedra caliza que se utiliza para la construcción; existe en abundancia en México.

8. Las investigaciones que se han realizado sobre el colegio fueron escritas por Enrique Olavarrìa y Ferrari, Gonzalo Obregón Jr., Josefina María Cristina Torales Pacheco y Josefina Muriel.

9. Accesoria de taza y plato es una habitación acondicionada como local comercial. En su interior cuenta con un tapango que le proporciona dos niveles. En la parte de abajo la persona que alquila el lugar coloca su negocio y la parte de arriba es utilizada como vivienda. Esta forma de alquiler brindaba comodidad y ahorro a la persona que rentaba estas accesorias porque no tenía que desplazarse a su lugar de trabajo y no debía cubrir dos rentas.

10. AMABPAC es Asociación Mexicana de Archivos y Bibliotecas Privadas, A.C. Para mayor información visitar su portal web: http://www.amabpac.org.mx

11. Muriel, 2006, p. 49.

12. Muriel, 2006, p. 58.

Corpus, Vol 3, No 2 | 2013 
13. Ripalda, 1863.

14. Fleury, 1884.

15. Loyola, 1999.

16. Algunos ejemplos de ello son: los hilvanes, el punto adelante o bastilla, el pespunte, el dobladillo, el punto por encima, la costura de faltriquera, la cruceta, los ojales, la cadeneta, el recogido o fruncido, la costura abierta, la vainica, entre otros.

17. Este podía realizarse con algunos pedazos cuadrados de muselina blanca de diferentes calidades, siendo preferible el algodón de color para trabajar, porque hacía la costura más visible que el algodón blanco y las faltas podían notarse, y podía acomodarse mejor en forma de libro, en tira o en un cuadrado grande.

18. Algunas de las puntadas del bordado que se elaboraban en Vizcaínas fueron las siguientes: el festón, el festón redondo con festoncitos, variante de la puntada anterior, el punto de cruz, el punto de rococó, pespuntes, punto cadeneta, pata de gallo, rellenos sueltos, rellenos lazados, punto inglés, punto pisano, el filtere, punto de cordoncillo, etc.

19. La puntada predominante en este estilo es el punto noruego. La puntada básica es el bloque kloster; este punto puede combinarse con otros como el ojal, el punto de cruz, la vainica, el punto de tallo, la cadeneta. El aprendizaje de este bordado es el grado máximo de dificultad en esta labor ya que en ella se deben dominar todas las puntadas y aplicaciones que se puedan poner en la pieza que se está realizando. El método utilizado para bordar es utilizando tela de cuadrillé, dividiéndola en cuatro partes iguales con un ligero hilván que puede ser con un hilo de color contrastado. Se continúa centrando los motivos, se cuentan los cuadros de la tela y se empiezan a bordar por el centro. Generalmente los primeros bordados son con un solo color de hilo y copiando dibujos o imágenes muy sencillas como podrían ser flores o el abecedario. Las piezas de bordado generalmente eran para objetos religiosos, cubre cálices, palias, los mantos para las vírgenes, manteles, hijuelas y sotanas para los capellanes. Para obtener mayor información consultar Atkinson (1908, pp. 135-136).

20. AHCV, E22 TI V16 “Constituciones del Colegio de San Ignacio de Loyola de México," constitución XXV.

21. Ibídem

22. Muriel, 2006, pp. 89-90.

23. Asimismo, se cuenta con material bibliográfico para la venta, que fue elaborado gracias a la consulta de las fuentes documentales del acervo. Estos son algunos de los títulos:

- Ana Rita Valero de García Lascuráin, Mors Vita, El panteón de Vizcaínas, México, Colegio de San Ignacio de Loyola Vizcaínas, 2007.

- Josefina Muriel, Los vascos en México y su colegio de las vizcaínas, México, Integración Editorial S.A. de C.V, 2006, segunda edición.

- Elvia Carreño Velázquez, Este amoroso tormento, el libro y la mujer novohispana, México, Colegio de San Ignacio de Loyola, Vizcaínas, ADABI, 2011.

- Julia Mörner, Memorias de una colegiala, México, Colegio de San Ignacio de Loyola, Vizcaínas

- Catálogo fondos bibliográficos Archivo Histórico "José María Basagoiti Noriega" del Colegio de San Ignacio de Loyola, Vizcaínas.

- Gaceta Vizcaínas, México, Colegio de San Ignacio de Loyola, Vizcaínas, No. 1-5 


\section{RESÚMENES}

Este artículo tiene como finalidad dar a conocer los acervos documentales que resguarda el Archivo Histórico "José María Basagoiti Noriega" del Colegio de San Ignacio de Loyola, Vizcaínas. La importancia de difundirlos es ofrecer a los lectores un acercamiento a la historia de México examinado a través del desarrollo de la vida de un colegio fundado por vizcaínos en la época novohispana. La institución logró adaptarse a las condiciones que le imponía ese contexto, y con ello consiguió continuar prestando sus servicios por más de cuatro siglos. Pretendemos que este material ofrezca nuevas alternativas para percibir la vida y desarrollo social, económico y político de la sociedad civil mexicana.

This article aims to present the documentary collections that protect the Historical Archive "Jose María Noriega Basagoiti" of the San Ignacio de Loyola College, Vizcaínas. It is important to spread this knowledge to provide readers an approach to the history of Mexico examined through the development of the life of a college founded by Basques in the era of New Spain. The institution was able to adapt to the new conditions imposed, and thus to continue serving for more than four centuries. This material is intended to offer new ways to perceive life and the social, economic and political development of Mexican civil society.

\section{ÍNDICE}

Keywords: Basques in Mexico, female education, College of San Ignacio de Loyola, Vizcaínas, Historical Archive

Palabras claves: vascos en México, educación femenina, Colegio de San Ignacio de Loyola, Vizcaínas, Archivo Histórico

\section{AUTORES}

\section{ANA RITA VALERO DE GARCÍA LASCURÁIN}

Directora del Archivo Histórico “José María Basagoiti Noriega”, del Colegio de San Ignacio de Loyola, Vizcaínas, México.

Correo electrónico: ana_rita@prodigy.net.mx

\section{NORA DEVEAUX CABRERA}

Comité de difusión de la Gaceta Vizcaínas, México.

Correo electrónico: nora_caux@hotmail.com 